\title{
Protéines et lipides : des technologies adaptées aux usages actuels et futurs
}

\section{Proteins and lipids: technologies for present ant future use}

Oléagineux, Corps Gras, Lipides. Volume 10, Numéro 1, 57-60, Janvier - Février 2003, Protéines et lipides végétaux : interactions nutritionnelles et fonctionnelles

Auteur(s) : Jacques EVRARD, CETIOM, Service Valorisation, qualité, sécurité sanitaire rue Monge, parc industriel, 33600 Pessac.

Author(s) : Jacques EVRARD

Summary : Oil and meal from rapeseed, sunflower and soya are classically used in food and feed and in non-food at a less extent. A particular case has to be underlined for soya, the protein fraction of which can be used in food as protein concentrates or protein isolates. In all these cases, oilseeds are processed by classical methods applied for many years without marked evolutions. Nevertheless, some trends are being observed in response to specific nutritional demands emerging from consumers with food safety concerns. New ways for valorization of oilseed end-products are being identified and their development is close conditioned by modification of classical processes or defining and setting of new flowsheets. Some significant projects are described in this paper.

Keywords : oilseeds, proteins, lipids, process, markets

\section{ARTICLE}

Les graines oléagineuses (colza, tournesol, soja) sont classiquement valorisées, au travers des technologies de la trituration et du raffinage, sous forme d'huiles utilisées en alimentation humaine (consommation directe et industrie alimentaire) et de tourteaux, sources de protéines, utilisés en alimentation animale (tableau 1). Des traitements complémentaires appliqués aux farines de soja permettent d'obtenir des concentrés et/ou des isolés de protéines utilisés en alimentation humaine, soit comme substituts de la viande, soit comme additifs apportant des propriétés fonctionnelles particulières.

Des demandes s'expriment actuellement pour faire évoluer et respecter des cahiers des charges mieux adaptés aux usages actuels des huiles et des tourteaux : exigences légitimes du consommateur non seulement pour les caractéristiques nutritionnelles des aliments, mais aussi en terme de sécurité sanitaire de ces aliments, qualité des aliments du bétail. Une volonté de diversification s'affirme par ailleurs, pour des produits à plus hautes valeurs ajoutées ; ce mouvement est notamment relayé par les industries de deuxième transformation.

Ces voies nouvelles de valorisation ne se conçoivent dans la plupart des cas qu'avec une évolution significative des technologies de transformation des graines oléagineuses. 
Le poids des technologies classiques

L'industrie de l'huilerie est relativement peu évolutive. Dans ses grandes lignes, le procédé de trituration est fixé depuis une cinquantaine d'années et dégage une faible valeur ajoutée. Les principaux progrès enregistrés au cours des dix dernières années ont notamment porté sur l'accroissement des débits (actuellement autour de $2000 \mathrm{t}$ de graines par jour pour du colza ou du tournesol, 5000 t pour le soja), sur les économies d'énergie et sur la réduction des rejets d'hexane et d'eaux usées.

L'examen des opérations unitaires nécessaires pour transformer les graines en huile et tourteaux (tableau 2), met en évidence le caractère particulièrement pénalisant de certaines d'entre elles pour la qualité, dans la mesure où elles mettent en ?uvre des températures élevées, ou plus généralement des traitements mécano-thermiques drastiques: traitement thermique avant pression mécanique des graines, désolvantation et toastage des tourteaux après extraction à l'hexane, traitement thermique au cours de la désodorisation des huiles durant le raffinage (tableau 3). Ces effets sont accentués dans le cas du colza dont les graines sont plus difficiles à triturer que d'autres: comportement irrégulier à la pression selon les lots de graines accompagné de problèmes à l'extraction, difficultés à désolvanter les tourteaux rendant nécessaires des traitements thermiques élevés.

Les conditions de trituration ont des répercussions non négligeables sur l'aptitude au raffinage des huiles.

Le raffinage permet d'éliminer les composés indésirables de l'huile brute, à savoir les phospholipides (démucilagination), les acides gras libres et les pigments (neutralisation, décoloration) et les composés odorants (désodorisation). L'optimisation du raffinage consiste à préserver les antioxydants naturels (tocophérols) et les acides gras polyinsaturés (AGPI) et à limiter les isomérisations cis-trans génératrices d'acides gras trans de même qu'à éliminer les éventuels résidus de pesticides. Ces objectifs de qualité nécessitent de trouver un compromis, notamment au niveau des conditions de température et de pression appliquées durant le raffinage classique. Il en résulte que tout procédé d'extraction de l'huile permettant d'alléger le raffinage nécessaire doit avoir des conséquences positives sur la qualité nutritionnelle de l'huile.

\section{L'évolution des technologies}

Quelques évolutions se dessinent cependant depuis quelques années. Elles répondent, soit à une demande directe des consommateurs (souhaits pour des huiles, classiques ou nouvelles, à haute valeur nutritionnelle), soit à une demande relayée par des industriels de seconde transformation, pour lesquels les arguments nutritionnels sont de puissants vecteurs de communication et de différenciation (par exemple des fabricants de biscuits, gros utilisateurs de corps gras ou utilisateurs de concentrés et d'isolés de protéines, manifestant leur intérêt pour une telle approche 
nutritionnelle en même temps qu'une sécurisation des approvisionnements), soit à une demande des fabricants d'aliments du bétail (qualité des tourteaux et traçabilité). Dans tous les cas, les marchés visés concernent les aliments, les alicaments ou la cosmétique.

En résumé, une volonté de progrès de la part des acteurs économiques de première et de deuxième transformation (triturateurs et formulateurs) s'affirme aujourd'hui ; elle prend en considération des problématiques nouvelles: production à cahiers des charges, huiles nouvelles, fabrication de concentrés de protéines, etc.. qui sont autant de facteurs favorables à une évolution significative des technologies. C'est ainsi que des procédés nouveaux sont (ou pourraient être) mis en lavre pour préserver les caractéristiques intrinsèques des graines (acides gras, composés mineurs des huiles, propriétés des protéines natives), modifier des propriétés (solubilité des protéines), éliminer des composés indésirables

Des procédés nouveaux

Des procédés présentés comme des alternatives à la pression et suivis ou non de l'extraction par solvant sont actuellement développés: l'extrusion-pression (Extrusion-expelling $\{E-E\}$ system), développée actuellement sur soja à I'Université d'Iowa [1], conduit à une farine de soja titrant $6 \%$ d'huile résiduelle; les propriétés fonctionnelles et les conditions d'incorporation de cette farine dans différents produits alimentaires sont en cours d'étude. D'autres travaux du même type sont menés par ailleurs [2,3]. L'extrusion à vis co-rotatives et cage de déshuilage dont le concept a été défini par I'UTC de Compiègne dès 1997, est actuellement développée à l'échelle pilote par le CETIOM, en collaboration avec l'atelier pilote d'huilerie CREOL. La société suédoise Karlshamns Crushing \& Feed $A B$ développe par ailleurs le système Expro qui consiste schématiquement à tanner les tourteaux par la chaleur (chauffage des flocons à $150^{\circ} \mathrm{C}$ pendant 20 secondes) en vue d'un usage à plus haute valeur ajoutée dans l'alimentation des ruminants par diminution de la dégradabilité de l'azote. Les gains revendiqués par l'application de ces nouvelles technologies concernent notamment la qualité des fractions protéiques (modulation de la solubilité) ainsi qu'une simplification du raffinage des huiles produites, autorisant en particulier le simple dégommage à l'eau.

Concernant le procédé ExPro, les revendications nutritionnelles sont résumées au tableau 4:

Deux programmes de recherche européens ont pris en compte ces voies de progrès avec l'objectif d'améliorer et de diversifier les usages des tourteaux de colza : le programme Europroteins, animé par le GIE EURETEC (1993-1999), a consisté à explorer les voies technologiques pour accroître les usages des tourteaux de colza en alimentation animale [4] et le programme Enhance (2000-2002) [5] dont la coordination est assumée par l'INRA de Nantes, consiste à identifier et à mettre au point de nouveaux usages des protéines de colza dans le domaine du non-alimentaire. 
Les solvants alternatifs

Le remplacement de l'hexane est motivé par des contraintes règlementaires (la limite admissible en trace d'hexane dans les huiles raffinées est passée de 5 ppm directive UE de 1990 à 1 ppm -directive UE de 1998 applicable depuis le 27 avril 1999 et 500 ppm pour les tourteaux au cours du transport) mais aussi par les cahiers des charges des consommateurs. Des solvants alternatifs tels que I'isohexane [6], l'éthanol (AL Bolier bv, Pays-Bas), l'iso-propanol (Food Protein R\&D Center, Texas University, EU) ou des fluides supercritiques sont en phase d'étude et de développement. La prise en compte de la demande d'authenticité toujours plus forte des consommateurs a conduit à utiliser l'eau comme solvant alternatif. L'eau n'étant pas un bon solvant de I'huile, le procédé d'extraction repose sur un principe différent qui consiste à créer un mélange en milieu aqueux de l'huile et des autres constituants de la graine et à séparer ceux-ci au moyen de techniques douces telles que la centrifugation. Bien que l'huile obtenue après raffinage soit de bonne qualité, l'objectif principal de l'extraction aqueuse est de préserver les propriétés nutritionnelles des protéines extraites. Les chercheurs sont donc à même de proposer des voies de transformation plus respectueuses des produits et de leurs qualités intrinsèques: moins de chocs mécanique et thermique, mise en ?uvre de process à basse température, pas d'utilisation de solvant organique.

Les techniques d'extraction à l'eau

De nombreux travaux d'extraction des huiles en milieu aqueux ont déjà été réalisés avec quelques variantes et sur plusieurs types de produits (colza, tournesol, soja, olive, avocat, palme) mais nombre d'entre eux n'ont pas dépassé le stade du laboratoire. En effet, la faible productivité de ces procédés en comparaison avec l'extraction à l'hexane n'a pas permis le développement d'applications commerciales $[7,8,9]$ Le problème de cette faible productivité peut être résolu en partie par I'utilisation d'enzymes hydrolytiques pour aider à la libération de I'huile [10-12]. Le procédé aqueux en présence d'enzymes a été exploré sur colza par Novo Industri A/S [13] : pré-traitement comprenant un broyage et un traitement thermique pour inhiber la myrosinase et les autres enzymes, traitement enzymatique pour dégrader les parois cellulaires et libérer l'huile, phases de séparation et séchage conduisant à 3 fractions (huile, farine riche en protéines, farine riche en sucres). L'optimisation de l'extraction assistée d'enzymes de différentes huiles a été étudiée à l'échelle du laboratoire [14-19]. Parmi les travaux qui ont été développés au stade pilote, on peut citer le procédé Friolex ${ }^{\circledR}$ (tableau 5) de Westfalia Separator GmbH [20] utilisation d'un mélange eaualcool qui a conduit sur colza à la production d'huile de haute qualité équivalente à de l'huile vierge de pression à froid et le procédé Bioraf (Danemark) avec pré-pression à froid et traitement enzymatique pour dégrader les parois cellulaires (tableau 6)

Ce dernier procédé, utilisé également sur colza dans le programme européen BOP (1998-2000) [21] pour la préparation de fractions protéiques à haute valeur ajoutée (protéines fonctionnelles valorisées en non-alimentaire), est toujours en phase d'expérimentation au stade pilote et semble davantage promis au développement d'usages non-alimentaires. 
En dehors des applications sur colza, il faut noter le procédé développé par ÖHMI Engineering GmbH (Magdebourg, Allemagne) pour le soja et qui consiste en un floconnage, un pré-traitement enzymatique en milieu aqueux, un séchage et une pression qui permet d'extraire $92 \%$ de l'huile. Le tourteau referme $52 \%$ de protéines, cette augmentation étant due à une élimination des polysaccharides hydrolysés et solubilisés par le milieu aqueux. La teneur résiduelle en huile du tourteau serait de l'ordre de 2 à $3 \%$. Bien que le procédé ne soit pas industrialisé, sa faisabilité est annoncée pour des unités traitant $200 \mathrm{t}$ de soja par jour.

En revanche, la technologie d'extraction en milieu aqueux est opérationnelle dans l'industrie de I'olive. Le fruit est broyé en milieu aqueux et l'huile est séparée par centrifugation. Ce même procédé a été testé pour l'extraction simultanée de l'huile de palme et de palmiste ([22], projet Drupalm) à partir du fruit de palme. Ce procédé a été développé par le CIRAD entre 1993 et 1994. Malgré une simplification de la technologie classique et l'obtention d'effluents plus faciles à traiter, le bilan économique s'est avéré négatif à cause de l'absence d'un positionnement technique et nutritionnel de cette huile à profil mixte d'acides gras.

Par ailleurs, la teneur en huile résiduelle de la fraction protéique dépend directement de la quantité de phosphatides présents dans l'huile. C'est ainsi qu'il est difficile d'obtenir des fractions protéiques de tournesol contenant moins de $6 \%$ d'huile [23].

Le raffinage des huiles brutes : un domaine d'innovations

Une grande attention est portée, depuis plusieurs années, sur l'optimisation du raffinage des huiles brutes avec l'objectif de répondre de mieux en mieux aux exigences des consommateurs tant au plan de la nutrition qu'au plan de la sécurité sanitaire. Le cahier des charges classique pour une huile raffinée est le suivant :

préservation des acides gras polyinsaturés,

absence d'isomères trans,

teneurs élevées en tocophérols,

absence de contaminants.

Concernant les contaminants, une attention particulière est portée sur les résidus éventuels de pesticides. La société De Smet [23] recommande des conditions de désodorisation relativement drastiques $\left(\mathrm{t}^{\circ} \mathrm{C}>230^{\circ} \mathrm{C}, \mathrm{p}<4 \mathrm{mbar}\right.$ ) pour une élimination efficace des pesticides, pratiques qui peuvent être contradictoires avec des contraintes de raffinage en conditions modérées. 
${ }^{1}$ - Voir OCL, 1997. Dossier : Evolution technologiques et corps gras, vol 4, Nº 1, p. 30-64.

\section{CONCLUSION}

L'adaptation de produits traditionnels aux besoins du marché ou la création de produits nouveaux impliquent la modulation des paramètres de la trituration classique et la mise au point de nouveaux diagrammes.

Cependant, les coûts induits par ces adaptations ne peuvent se justifier que par une plus-value acceptable des produits, l'évolution de contraintes réglementaires, d'environnement ou le respect de cahiers des charges notamment en ce qui concerne la sécurité sanitaire des aliments.

\section{REFERENCES}

1 - Deland J Myers, Adrianna A. Characterization and utilization of soy flour produced from extrusionexpelling processing. Inform 2002 ; vol. 13, n 9, p. 708-13.

2 - Caetano MF, Couri S, Freitas SP (2002). Extraction aqueuse enzymatique de l'huile de tournesol extrudé. Riv Ital Sostanze Grasse ; vol. 79, n 5, p. 165-9.

3 - Freitas SP, Hartman L, Couri S. Application combinée de l'extrusion et de la technologie enzymatique pour l'extraction de l'huile de soja. Fett-lipid 1997 ; vol. 99, nº 9, p. 333.

4 - Le Guen MP. Optimisation de l'utilisation en alimentation animale des oléoprotéagineux européens, phase de recherche EURETEC 93/99 (rapport final, non publié) 1999.

5 - Enhance, nº QLK5 1999 - 01442, Green chemicals and biopolymers from rapeseed meal with enhanced end-uses performance, 2nd Progress report, 1-02-01 to 31-01-02 (confidential).

6 - Inform, Isohexane: likely choice for crushers seeking to replace $n$-hexane? 2002 ; vol. $13, n^{\circ} 4, p$. 282-6.

7 - Ohlson R. Technologie récente de l'huile de colza. J Am Oil Chem Soc 1992 ; vol. 69, n 3, p. 195-8.

8 - Eapenk E, Kalbag SS, Subrahmanyan V. Operations in wet-rendering of peanut for the separation of protein, oil, and starch. J Am Oil Chem Soc 1966 ; 43, p. 585-9. 
9 - Hagenmaier RD, Cater CM, Mattil KF. Aqueous processing of fresh coconuts for recovery of oil and coconut skim milk. J Food Sci 1973 ; 54, p. 491-2.

10 - Sineiro J, Dominguez $\mathrm{H}$, Nunez MJ. Influence du traitement enzymatique sur la qualité des huiles végétales. Grasas Aceites 1998A ; vol. 49, n² 2, p. 191-202.

11 - Sineiro J, Dominguez H, Nunez MJ. Optimisation du traitement enzymatique pendant l'extraction aqueuse de l'huile de graine de tournesol. Food Chem 1998b ; vol. 61, n4, p. 467-74.

12 - Rosenthal A, Pyle DL, Niranjan K. Aqueous and enzymatic processes for edible oil extraction. Enz Microb Technol 1996 ; 19, p. 402-20.

13 - Christensen M. Extraction by aqueous enzymatic processes. Inform 1991 ; vol. 2, nº 11, p. 984-7.

14 - Aparna Sharma, Khare SK, Gupta MN. Enzyme-assisted aqueous extraction of peanut oil, JAOCS ; 2002: vol. 79, $n^{\circ} 3$, p. 215-8.

15 - Badr FH, Sitohy MZ. Conditions optimales pour l'extraction de l'huile de tournesol. Grasas Aceites 1992 ; vol. 43, n 5, p. 281-3.

16 - Bouvier F. Utilisation de cellulases et pectinases dans le procédé d'extraction d'huile de palme. Revue Française des Corps Gras 1991 ; vol. 39, p. 245-52.

17 - Sarkar BC, Singh BPN, Agrawal YC et al.. Optimisation du prétraitement enzymatique pour augmenter le rendement en huile. J Food Sci 1998 ; vol. 35, nº 2, p. 183-6.

18 - Sitohy MZ, Badr EH, Perifanova-Nemska M. Caractérisation de l'huile de tournesol extraite enzymatiquement ainsi que les résidus protéiques. Grasas Aceites 1993 ; vol. 44, nº 6, p. 345-47.

19 - Sosulski K, Sosulski FW. Technologie de l'huile de canola par traitement enzymatique ou voie classique : qualité des produits et évaluation des coûts. J Am Oil Chem Soc 1993 ; vol. 70, nº 9, p. 8259.

20 - Hruschka S, Frische R. A new oil extraction process. OCL 1998 ; vol. 5, n 5, p. 356-60.

21 - BOP. Biorefining Oilseed Proteins. FAIR $2000 ; 1998-2000$. 
22 - Noel JM, Rouziere A, Graille J, Pina M. Procédé DRUPALM : du nouveau pour les huileries de palme. OCL 1997 ; vol 4, n 1, p. 38-42.

23 - Lusas E, Watkins LR, Rhee KC. Separation of Fats and Oils by Solvent Extraction: Non-Traditional Methods, in Edible Fats and Oils Processing Basic Principles and Modern practices ; 1990 : p. 56-80.

24 - (W. De Greyt, 2001)

25 - Arnaud G. Procédé et dispositif pour l'extraction de l'huile que contiennent certains végétaux, Brevet France, FR $2718459 ; 1994$.

26 - Bucher-Guyer AG. Procédé et appareillage pour extraire les corps gras de produits naturels, Brevet Monde, WO $8909255 ; 1989$.

27 - Génie Industriel Alimentaire. Techniques séparatives. Tome II. Lavoisier, Paris, 1992.

28 - OCL. Dossier : Évolution technologiques et corps gras 1997 ; vol 4, n 1, p. 30-64.

29 - Soren Marcus Pedersen, Morten Gylling. The economics of producing quality oils, proteins and bioactive products for food and non-food purposes based on biorefining, Ministeriet for Fodevarer, Landbrug og Fiskeri, Working paper ; 2001.

Illustrations

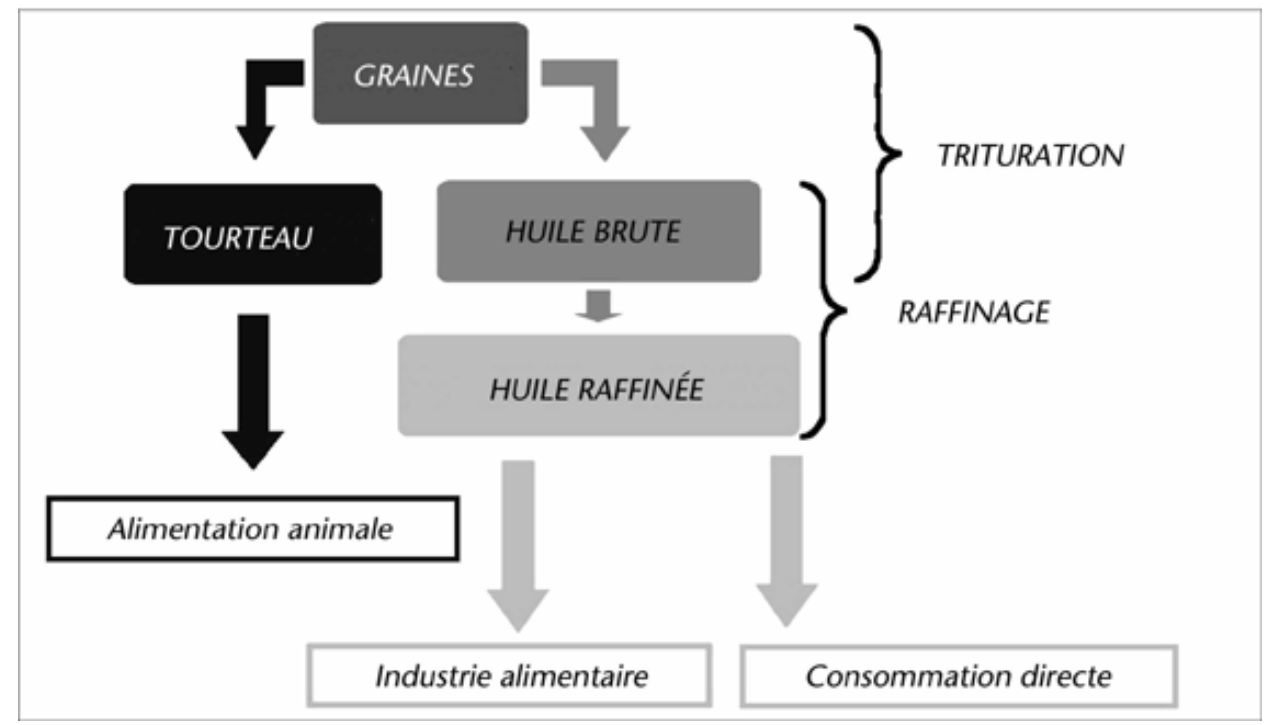

Tableau 1 - Valorisation des graines oléagineuses 


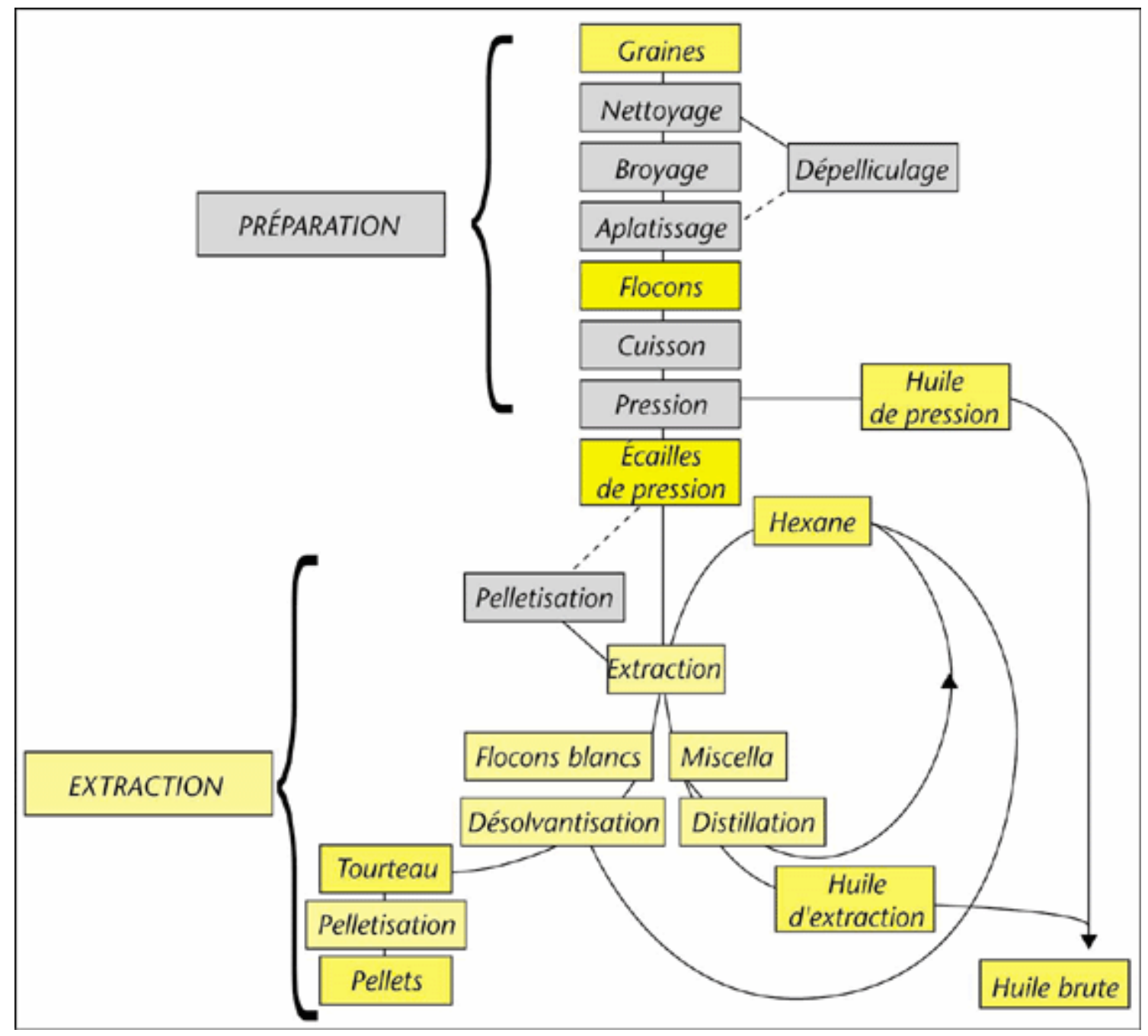

Tableau 2 - Diagramme classique de la trituration 


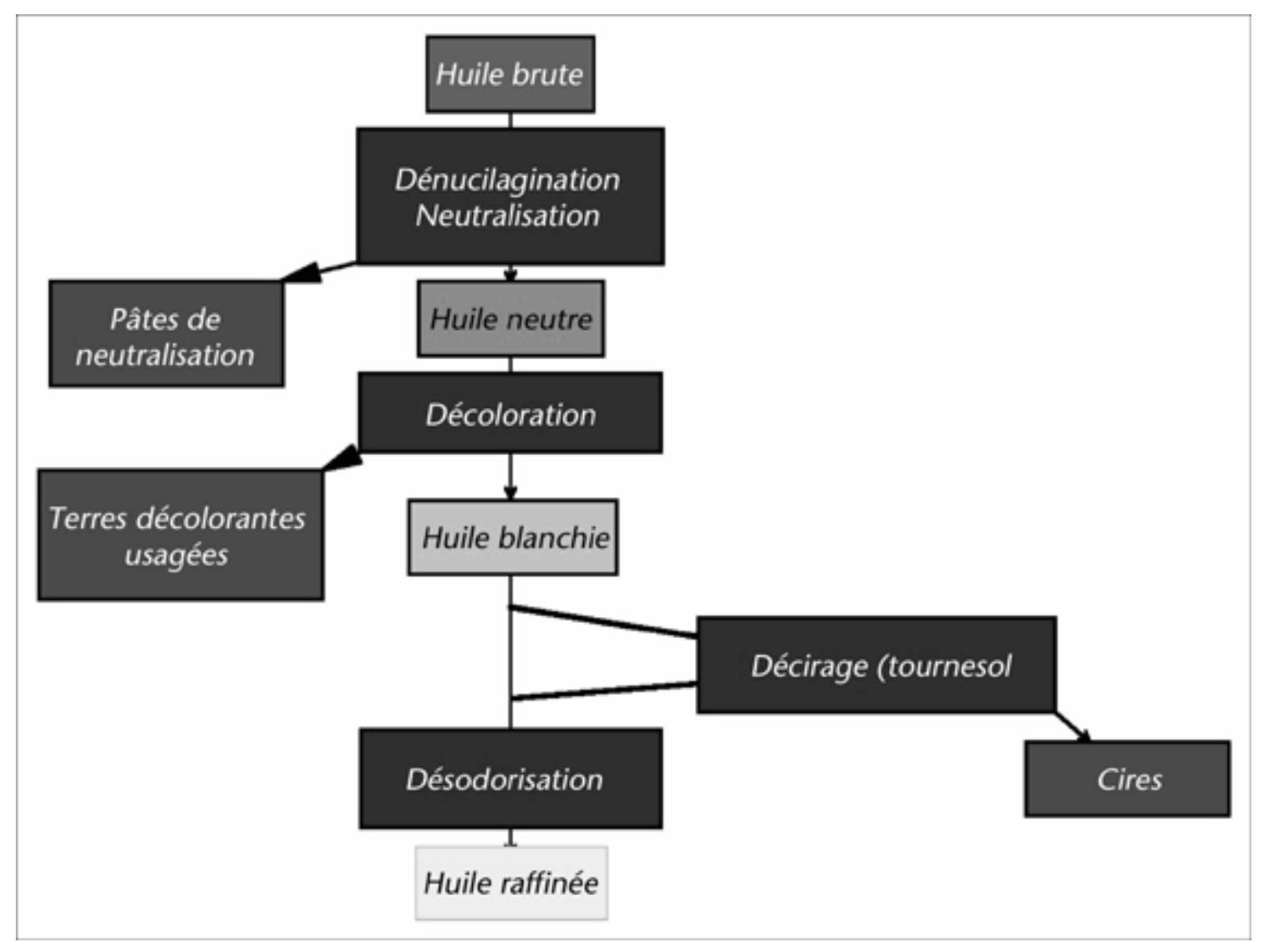

Tableau 3 - Diagramme classique du raffinage.

\begin{tabular}{|lcc}
\hline & Tourteau de colza normal & Tourteau de colza ExPro \\
\hline Matières azotées totales (\%MS) & 38,3 & 38,2 \\
Solubilité dans la salive (\%MAT) & 24,6 & 8,7 \\
Solubilité dans la pepsine (\%MAT) & 87,5 & 86,9 \\
Dégradabilité de l'azote (\%) & 72 & 46 \\
Digestibilité de l'azote (\%) & 82 & 82 \\
\hline
\end{tabular}

Tableau 4 - Tannage et valeur alimentaire du tourteau de colza ExPro. 
- Broyage puis maturation dans un mélange eau-alcools

- Récupération de I'huile par centrifugation

- Séchage de l'huile et du tourteau

- Recyclage des alcools

- Travail sous azote et à basse température $\left(\max .70^{\circ} \mathrm{C}\right)$

- Rendement d'extraction voisin de $95-98 \%$

Revendications

- Huiles de haute qualité (peu colorée, préservation des tocophérols et acides gras polyinsaturés)

équivalente à une huile vierge de pression à froid

- Raffinage simplifié

Tableau 5 : Principales caractéristiques du procédé Friolex ${ }^{\circledR}$

Concept de « bioraffinage des produits agricoles

- Dépelliculage et prépression à froid - Traitement enzymatique en milieux aqueux (dégradation des parois cellulaires) - Séparation de l'huile par centrifugation - Fractionnement du co-produit pour isoler des composés à haute valeur ajoutée - Pas d'industrialisation annoncée

Tableau 6 - Principales caractéristiques du procédé Bioraf. 